\title{
Attentional networks and consciousness
}

\author{
Michael I. Posner* \\ Department of Psychology, University of Oregon, Eugene, OR, USA
}

\section{Edited by:}

Naotsugu Tsuchiya, Monash

University, Australia

Reviewed by:

Anil K. Seth, University of Sussex, UK Ryota Kanai, University College

London, UK

\section{*Correspondence:}

Michael I. Posner, Department of Psychology, University of Oregon, 1254, 222 Huestis Hall, Eugene, OR 97403-1254, USA.

e-mail:mposner@uoregon.edu
The term consciousness is an important one in the vernacular of the western literature in many fields. It is no wonder that scientists have assumed that consciousness will be found as a component of the human brain and that we will come to understand its neural basis. However, there is rather little in common between consciousness as the neurologist would use it to diagnose the vegetative state, how the feminist would use it to support raising male consciousness of the economic plight of women and as the philosopher would use it when defining the really hard question of the subjective state of awareness induced by sensory qualities. When faced with this kind of problem it is usual to subdivide the term into more manageable perhaps partly operational definitions. Three meanings that capture aspects of consciousness are: (1) the neurology of the state of mind allowing coherent orientation to time and place (2) the selection of sensory or memorial information for awareness and (3) the voluntary control over overt responses. In each of these cases the mechanisms of consciousness overlap with one or more of the attentional networks that have been studied with the methods of cognitive neuroscience. In this paper we explore the overlap and discuss how to exploit the growing knowledge of attentional networks to constrain ideas of consciousness.

Keywords: attention networks, alerting, orienting, executive

\section{INTRODUCTION}

A previous paper on this topic Posner (1994) argued that the mechanisms of attention form the basis for an understanding of consciousness. Since that time the study of attention has greatly advanced (Petersen and Posner, 2012; Posner, 2012). While the intervening years have provided evidence of dissociations between brain networks involved in attention and aspects of consciousness (Koch and Tsuchiya, 2007), I still believe that much can be learned about consciousness from an understanding of attention.

As many have pointed out there are dissociations between attention and consciousness, however, there are also dissociations between various aspects of attention and some of the latter may help account for the former.

In this paper I first summarize the relation of attention and consciousness and illustrate how the study of attentional networks might help illuminate dissociations. Because attention involves different brain networks (Posner and Petersen, 1990; Posner and Rothbart, 2007) and because consciousness has a wide variety of definitions it is necessary to illustrate their constraints and inter-relations rather than provide a single unified account. I try to do this by dealing first with the conscious state, second with consciousness of sensory qualities and finally with volition. The distinction between conscious state and content is one that has been frequently made (Laureys, 2005). The idea that there is a level of voluntary control or will that reflects conscious control of behavior is also a common idea (Baumeister et al., 2007). It turns out that each of these definitions is predominantly associated with a different attentional network. I then turn more briefly to the issue of the most important unsolved questions and the methods that their solution might require.

\section{STATE}

Within neurology consciousness often refers to a brain state in which the person is capable of responding to external events and relate them to the self (Posner et al., 2007a). This state is closely associated with the concept of arousal and to the diurnal cycle of sleep and wake. Clearly during sleep we are unable to respond appropriately to many external events and patients with lesions of the arousal system are often unable to determine their current location or the time of day or year.

Recent fMRI studies have revealed that the resting brain involves activity in two oscillating brain networks (Raichle, 2009). In experiments it is possible to provide a warning signal that a target is about to occur. The participant relaxes between trials into a state which is toward that obtained during continued rest, the warning signal alters this state. These studies have revealed the importance of the brain's norepinephrine system arising in the brain stem locus coeruleus and involving both frontal and parietal brain areas. In the posterior part of the brain this system influences more dorsal areas involved in orienting of attention toward sensory stimuli and has little direct input into strictly sensory areas of vision. A warning signal too fleeting to be conscious can still induce the alert state. Here is one of the places where a state change related to attention provides a critical bridge for awareness. If being alert is called paying attention than one can say attention can occur without consciousness of the signal that caused it.

By having a person to participate in a long and somewhat dull task one can measure their vigilance over time (Posner and Petersen, 1990; Posner, 2008, 2012). The right cerebral hemisphere is most involved in maintaining the alert state over long periods of time (tonic alertness), while the left cerebral hemisphere responds more to phasic changes induced by warning signals. Support for 
this link between the mechanisms of alertness and the conscious state comes from a recent finding showing that the conscious state of rats can be eliminated by injecting anesthetic into the midpontine tegmental system which has close connections to the locus coeruleus, the anterior cingulate, and other frontal structures (Sukhotinsky et al., 2007). Thus detailed findings about the physiology of the alert state provide a background for the study of consciousness.

Even in the absence of the alert state we can be conscious of internal events as in dreams. In the vegetative state there is evidence of arousal but no evidence of consciousness, showing that while the alert state clearly influences consciousness it is not sufficient for awareness of external events.

\section{SENSORY AWARENESS}

An important distinction in studies of awareness (Iwasaki, 1993) is between general knowledge of our environment (ambient awareness) and detailed focal knowledge of a scene (focal awareness). We generally believe that we have full conscious awareness of our environment, even when our focal attention is upon our own internal thoughts. Experimental studies (Rensink et al., 1997), show us how much this opinion is in error. In the study of "change blindness" when cues that normally lead orienting of attention are suppressed, we have only a small focus for which we have full knowledge and even major semantic changes in the remainder of the environment are not reported.

Change blindness is closely related to studies of visual search which have been prominent in the field of attention and are known to involve an interaction between information in the ventral visual pathway about the object identity and information in the dorsal visual pathway that controls orienting to sensory information (for a review, see Driver et al., 2004). Visual search tasks have been important for examining what constraints attention provides to the nature of our awareness of a target event. There is clear evidence that attention to a visual event increases the brain activity associated with it. Most evidence arises from studies using event related electrical potentials with visual stimuli and these have clearly shown that early sensory components of the visual evoked potential P1 and N2 (80-150 ms) are enlarged by the presence of attention (Hillyard et al., 2004).

If attention can serve to constrain conscious experience its presence indicated by enhanced $\mathrm{P} 1$ should increase the perceived luminance of a visual target and lead to the judgment that it is brighter than would otherwise been reported. Indeed Yeshurun and Carrasco (1999) found that a cue that causes orienting to a target can serve to changes its contrast function. Moreover, the increased P1 found in the EEG studies would support also support this prediction. However, this shift in visibility when orienting to a visual stimulus apparently does not occur, at least for stimuli that are sufficiently bright to be conscious. In an extensive series of experiments Prinzmetal et al. (1997) have shown that being able to pay attention to a stimulus or directing attention to a stimulus location reduces the variability of judgments about luminance or other stimulus dimensions but does not produce a subjective brightening of the stimulus. This suggests an important dissociation between luminance increases and attention on subjective experience even when they influence the same component of the scalp recorded ERP. While it is possible that a previously unseen stimulus will become conscious when attended, it appears generally that top down influence on sensory systems can usually be distinguished from sensory changes even when they involve the same general neural systems.

In a review of the literature Koch and Tsuchiya (2007) argued that attention and consciousness of external stimuli are two distinct brain processes. They produce a fourfold table that distinguishes between non-conscious and conscious processes and between attended and non-attended. They argue that all four possibilities are possible. Two of them, attention without consciousness and consciousness without attention, are dissociations. For example, they argue that the pop out effect in visual search, or the ability to provide the gist of a scene are examples of conscious processes that can occur without attention. This idea seems to confuse orienting of attention to items in the display, which is often not reported, with focal attention involved in processing the target. Duncan (1980) showed that multiple locations can be monitored simultaneously, but that the detection of one target drastically reduces the ability to detect another target. This reflects the important distinction that orienting can be summoned very efficiently to a target if it is not currently engaged but once engaged it is difficult to disengage. Corbetta and Shulman (2002) have shown that the temporal parietal junction of the right hemisphere is critical brain area for disengaging the orienting mechanism. This mechanism imposes a strong limitation on orienting to multiple objects.

Koch and Tsuchiya (2007) also argue that attention is required for priming, visual search, or thought but these things may not give rise to consciousness. The issue of whether attention is needed for priming illustrates how attention is not unified. Priming can occur even when no attention is allocated to the meaning of the prime. For example, a bias to treat the word "palm" as a tree does not eliminate the priming of finger at least for a brief time (Marcel, 1983). This shows that attention in one sense is not needed for priming. However, if attention is allocated to a letter the ability of the word of which the letter is a part to improve processing of related words (semantic priming) is reduced or eliminated. Moreover, the influence of a prime appears to depend on the person allocating attention at the proper time and place (Naccache et al., 2002; Dehaene, 2004), even if unaware that a word was presented. As the exact mechanisms of priming are better understood we may learn more about how the form of attention involved relates to its subjective experience.

Consider the process of developing the next sentence for this paper. No one would be surprised if a knock on the door to which I oriented interrupted my work and led to forgetting my place. One could argue that increased attention (in this case to the door) diminished awareness of the words of my sentence. Is this an instance of increased attention leading to decreased awareness? No one would really accept that. Rather they would say that I was simply attending to the wrong thing. However, similar confusions do infect the literature.

An example of confusion between attention networks arises in the opposite effects of attention and awareness on the duration of afterimages (van Boxtel et al., 2010; Murd and Bachmann, 2011). These experiments involve a full factorial design in which attention to the target is manipulated by a dual task while visibility is manipulated by the presence of a suppressor stimulus in the opposite eye. This is a very clever experimental design, but the interpretation of a 
dissociation between attention and visibility (consciousness) may not be correct. There is much evidence that dual tasks involve a common bottleneck (Sigman and Dehaene, 2008; Tombu et al., 2011) and that frontal areas common to the executive attention network are involved (Petersen and Posner, 2012). Thus dual tasks would reduce the availability of the executive attention system, but its role on convert orienting to the Gabor inducer is simply not defined. While viewing attention as one unified thing leads one to conclude that attention is reduced the network view says only the executive attention is reduced while orienting is simply not controlled. Suppose orienting was similar regardless of the difficulty of the dual task. The afterimage should be the same. Why is there a small increase of the duration of the afterimage during the difficult dual task condition. I really do not know the answer to this, but it could have to do with fatigue of the executive system producing a small slowing of response time to the reported image (see Baumeister et al., 2007).

This may not be the correct explanation of the experiment but it illustrates how different attention networks are involved in this experiment. Reporting the length of the visual aftereffect depends on the ability of the executive system, including the anterior cingulate, anterior insula and other frontal and basal ganglia structures to produce a report of the contents of consciousness. However, determining the direction or location of a target event involves the orienting system, including the inferior and superior parietal lobe, and frontal eyefields. Not only are these networks anatomically separate, but they are poorly correlated in performance. The finding that they are negatively related during afterimages is certainly interesting and important, but it does not raise any fundamental problem for the relevance of attention networks to understanding conscious reports.

Perhaps even more striking evidence is a study (Olivers and Nieuwenhuis, 2005), in which observers report the second of two rapidly presented stimuli more often when they are distracted by an another task than when they are fully concentrated on the display. This shows that a reduction of attention can be accompanied by an increase in awareness of the target. This striking demonstration may reflect different attentional networks. The attentional blink arises as the person concentrates on target 1 and as a consequence target 1 and target 2 are not perceived as separate events. The dramatic dissociation between awareness of a target and the availability of attention provides evidence that the two phenomena are not exactly the same. This dissociation may arise because there is a specific inhibition to processing a second target when it is similar to a first target which is still being attended. If concentration on the first target is reduced by a second task the inhibition may be released. These inhibitions are common in studies of attention, for example, in visual search orienting to a location slows reorienting to that same location (inhibition of return). If a second target is inhibited when an identical or related first target is focally attended, the dual task would reduce focal attention on the first target and lead to the paradoxical improved performance on the second target. Divided attention would provide a way of reducing overall focal attention thus leading to the observed improvement in performance.

\section{VOLITION}

Focal attention to the target of a visual search appears to involve an executive attention network that includes the anterior cingulate, anterior insula, lateral prefrontal areas, and the underlying basal ganglia (Posner and Rothbart, 2007). Humans have a conviction of conscious control that allows us to regulate our thoughts, feelings, and behaviors in accord with our goals and people believe that voluntary conscious choice guides at least a part of the action we take. These beliefs have been studied under various names in different fields of psychology. In cognition, cognitive control is the usual name for the voluntary exercise of intentions, while in developmental psychology many of the same issues are studied under the name self-regulation and in the common term willpower (Posner et al., 2007b; Baumeister and Tierney, 2011).

Imaging studies suggest that whenever we bring to mind information, whether extracted from sensory input or from memory, we activate the executive attention network (Posner and Petersen, 1990; Posner, 2012). This may be because focal attention is voluntarily switched to the target information. Thus moving attention to a target in order to bring it fully to mind is one type of voluntary response. As such it has been hypothesized that it would require the executive attention network irrespective of the source of information (Posner and Petersen, 1990; Petersen and Posner, 2012).

We started out this paper with the traditional distinction between awareness and control as components of consciousness. However, one form of awareness, focal awareness, appears to involve the same underlying mechanism as involved in control. In this sense even though some forms of consciousness (e.g., ambient awareness) may have diverse sources within sensory specific cortex, there is also a degree of unity of the underlying mechanism involved in some aspects of consciousness (e.g., focal awareness and voluntary control). The distinction between focal and ambient factors in consciousness has been made before (Iwasaki, 1993) and it may help to clarify the sense of awareness that can be present even when detailed accounts of the scene are not possible as in change blindness (Rensink et al., 1997).

\section{ISSUES AWAITING SOLUTION}

The study of attention has made great strides in the last several years. It has been possible to combine imaging, genetics, and even cellular studies in humans, monkeys, and rodents to examine aspects of networks involved in the various functions of attention (Posner and Rothbart, 2007; Posner, 2012).

One way to proceed involves continuing the development of models of attention. We can then determine the constraints upon various definitions of consciousness they might provide. We need also to keep in mind that in the end these constraints may not be sufficient to entirely answer the many issues related to consciousness. It is important to realize that mapping of attention and consciousness is not one to one, but rather a mapping that involves several attentional functions or networks in addition to the several meanings of consciousness.

In many situations there is a strong correlation between orienting to and detecting a target. For example, a target at an attended location is more likely and faster to be reported. However, the separate RT subtractions of the Attention Network Test often yield low or no correlation between the orienting, alerting, and executive networks (Fan et al., 2002, 2009). In addition the networks have been shown to be separate anatomically in functional MRI studies (Fan et al., 2005), they involve different white matter tracts (Niogi 
and McCandliss, 2009), separate neurotransmitters (Marrocco and Davidson, 1998), different oscillator frequencies (Fan et al., 2007), and depend on different genetic polymorphisms (Green et al., 2008). These separations between attentional networks may make the report of differences between attention and awareness in brain oscillations (Wyart and Tallon-Baudry, 2008) less surprising since different forms of attention also give rise to different oscillations (Fan et al., 2007).

Some additional avenues for exploring the relationship between attention and consciousness may involve how altered states such as those induced by brain injury, hypnotism, drugs, or meditation

\section{REFERENCES}

Baumeister, R. F., and Tierney, J. (2011). Willpower: Rediscovering the Greatest Human Strength. New York: Penguin Group.

Baumeister, R. F., Vohs, K. D., and Tice, D. M. (2007). The strength model of self control. Curr. Dir. Psychol. Sci. 16, 351-355.

Corbetta, M., and Shulman, G. L. (2002). Control of goal-directed and stimulus-driven attention in the brain. Nat. Rev. Neurosci. 3, 201-215.

Dehaene, S. (2004). "The neural bases of subliminal word priming and conscious word recognition," in Attention and Performance XX: Functional Brain Imaging of Visual Cognition, eds N. Kanwisher and J. Duncan (Oxford: Oxford University Press), 205-224.

Driver, J., Eimer, M., and Macaluso, E. (2004). "Neurobiology of human spatial attention: modulation, generation, and integration," in Attention and Performance XX: Functional Brain Imaging of Visual Cognition, eds N. Kanwisher and J. Duncan (Oxford: Oxford University Press), 267-300.

Duncan, J. (1980). The locus of inerference in the perception of simultaneous stimuli. Psychol. Rev. 87, 272-300.

Fan, J., Byrne, J., Worden, M. S., Guise, K. G., McCandliss, B. D., Fossella, J., and Posner, M. I. (2007). The relation of brain oscillations to attentional networks. J. Neurosci. 27, 6197-6206.

Fan, J., Gu, X., Guise, K. G., Liu, X., Fossella, J., Wang, H., and Posner, M. I. (2009) Testing the behavior interaction and integration of attentional networks. Brain Cogn. 70, 209-220.

Fan, J., McCandliss, B. D., Fossella, J., Flombaum, J. I., and Posner, M. I. (2005). The activation of attentional networks. Neuroimage 26, 471-479.

Fan, J., McCandliss, B. D., Sommer, T., Raz, M., and Posner, M. I. (2002). Testing the efficiency and independence of attentional networks. J. Cogn. Neurosci. 3, 340-347.

Green, A. E., Munafo, M. R., DeYoung, C. G., Fossella, J. A., Fan, J., and Gray,
J. A. (2008). Using genetic data in cognitive neuroscience: from growing pains to genuine insights. Nat. Rev. Neurosci. 9, 710-720.

Hillyard, S. A., Di Russo, F., and Martinez, A. (2004). "The imaging of visual attention," in Attention and Performance XX: Functional Brain Imaging of Visual Cognition, eds J. Duncan and N. Knwisher (Oxford: Oxford University Press), 381-390.

Iwasaki, S. (1993). Spatial attention and two modes of visual consciousness. Cognition 49, 211-233.

Koch, C., and Tsuchiya, N. (2007). Attention and consciousness: two distinct brain processes. Trends Cogn. Sci. (Regul. Ed.) 11, 16-22.

Laureys, S. (2005). The neural correlate of (un)awareness: lessons from the vegetative state. Trends Cogn. Sci. (Regul. Ed.) 9, 556-559.

Marcel, A. J. (1983). Conscious and unconscious perception: experiments on visual masking and work recognition. Cogn. Psychol. 15, 197-237.

Marrocco, R. T., and Davidson, M. C. (1998). "Neurochemistry of attention," in The Attentive Brain, ed. R. Parasuraman (Cambridge, MA: MIT Press), 35-50.

Murd, C., and Bachmann, T. (2011). Spatially localized motion aftereffect disappears faster from awareness when selectively attended to according to its direction. Vision Res. 51, 1157-1162.

Naccache, L., Blandin, E., and Dehaene, S. (2002). Unconscious masked priming depends on temporal attention. Psychol. Sci. 13, 416-424.

Niogi, S., and McCandliss, B. D. (2009). Individual differences in distinct components of attention are linked to anatomical variations in distinct white matter tracts. Front. Neuroanat. 4:2. doi:10.3389/neuro.05.002.2010

Olivers, C. N. L., and Nieuwenhuis, S. (2005). The beneficial effect of concurrent task-irrelevant mental activity on temporal attention. Psychol. Sci. 16, 265-269. that change attention vary the quality of our conscious experience. The study of each of these states has been enhanced by the use of neuroimaging both of gray matter areas and of the connectivity between activated brain areas. Real time analysis of this connectivity will probably be crucial to the full specification of the networks of attention and of consciousness (Posner et al., 2006; Raichle, 2009).

\section{ACKNOWLEDGMENTS}

This paper was supported by NICHD grant HD060563 to Georgia State University.

Petersen, S. E., and Posner, M. I. (2012). The attention system of the human brain: 20 years after. Ann. Rev. Neurosci. 35 , (in press).

Posner, J. B., Saper, C. B., Schiff, N., and Plum, J. B. (2007a). Plum and Posner's Diagnosis of Stupor and Coma. New York: Contemporary Neurology.

Posner, M. I., Rothbart, M. K., Sheese, B. E., and Tang, Y. (2007b). The anterior cingulate gyrus and the mechanisms of self regulation. J. Cogn. Affect. Soc. Neurosci. 7, 391-395.

Posner, M. I. (2008). "Measuring alertness," in Molecular and Biophysical Mechanisms of Arousal, Alertnes and Attention, eds D. W. Pfaff and B. L. Kieffer (Boston: Blackwell), 193-199.

Posner, M. I. (1994). Attention: the mechanism of consciousness. Proc. Natl. Acad. Sci U.S.A. 91, 7398-7402.

Posner, M. I. (2012). Attention in the Social World. New York: Oxford University Press.

Posner, M. I., and Petersen, S. E. (1990). The attention system of the human brain. Annu. Rev. Neurosci. 13, 25-42.

Posner, M. I., and Rothbart, M. K. (2007). Research on attention networks as a model for the integration of psychological science. Annu. Rev. Psychol. 58, 1-23.

Posner, M. I., Sheese, B, Odludas, Y., and Tang, Y. (2006). Analyzing and shaping neural networks of attention. Neural. Netw. 19, 1422-1429.

Prinzmetal, W., Nwachuku, I., Bodanski, L., Blumfeld, L., and Shmuzu, N. (1997). The phenomenology of attention 2. Brightness and contrast. Conscious. Cogn. 6, 372-412.

Raichle, M. E. (2009). A paradigm shift in functional brain imaging. J. Neurosci. 29, 12729-12734

Rensink, R. A., O’Regan, J. K., and Clark, J. J. (1997). To see or not to see: the need for attention to perceive changes in scenes. Psychol. Sci. 8, 368-373.

Sigman, M., and Dehaene, S. (2008). Brain mechanisms of serial and parallel processing during dual task performance. J. Neurosci. 28, 7585-7598.

Sukhotinsky, I., Zalkind, V., Lu, J., Hopkins, D. A., Saper, C. B., and Devor, M. (2007). Neural pathways associated with loss of consciousness caused by intracerebral microinjection of GABA- active anesthetics. Eur. J. Neurosci. 25, 1417-1436.

Tombu, M. N., Asplund, C. L., Dux, P. E., Godwin, D., Martin, J. W., and Marois, R. (2011). Unified attentional bottleneck in the human brain. Proc. Natl. Acad. Sci. U.S.A. 108, 33.

van Boxtel, J. J., Tsuchiya, N., and Koch, C. (2010). Opposing effects of attention and consciousness or afterimages. Proc. Natl. Acad. Sci. U.S.A. 107, 8883-8888.

Wyart, V., and Tallon-Baudry, C. (2008). Neural dissociation between visual awareness and spatial attention. J. Neurosci. 28, 2667-2679.

Yeshurun, Y., and Carrasco, M. (1999). mance in spatial resolution tasks. Vision Res. 39, 293-306.

Conflict of Interest Statement: The author declares that the research was conducted in the absence of any commercial or financial relationships that could be construed as a potential conflict of interest.

Received: 29 August 2011; accepted: 18 February 2012; published online: 12 March 2012.

Citation: Posner MI (2012) Attentional networks and consciousness. Front. Psychology 3:64. doi: 10.3389/fpsyg.2012.00064

This article was submitted to Frontiers in Consciousness Research, a specialty of Frontiers in Psychology.

Copyright (c) 2012 Posner. This is an open-access article distributed under the terms of the Creative Commons Attribution Non Commercial License, which permits non-commercial use, distribution, and reproduction in other forums, provided the original authors and source are credited. Spatial attention improves perfor- 